\title{
Data S3
}

\section{Candidate reference genes for mRNA normalization}

\section{BestKeeper version 1}

http://gene-quantification.com/bestkeeper.html

liver

\begin{tabular}{|c|c|c|c|c|c|c|c|}
\hline \multicolumn{8}{|c|}{ CP data of housekeeping Genes: } \\
\hline & HPRT1 & B2M & $18 S$ & RPIP0 & GAPDH & АСТВ & HMBS \\
\hline & HKG 1 & HKG 2 & HKG 3 & HKG 4 & HKG 5 & HKG 6 & HKG 7 \\
\hline $\mathbf{n}$ & 11 & 11 & 11 & 11 & 11 & 11 & 11 \\
\hline geo Mean [CP] & 21,46 & 14,15 & 23,68 & 18,44 & 17,86 & 18,88 & 23,34 \\
\hline ar Mean $[\mathrm{CP}]$ & 21,47 & 14,16 & 23,69 & 18,45 & 17,88 & 18,92 & 23,36 \\
\hline $\min [C P]$ & 20,68 & 13,19 & 22,77 & 17,17 & 16,74 & 17,15 & 22,04 \\
\hline $\max [C P]$ & 22,46 & 14,79 & 25,12 & 19,62 & 20,51 & 21,67 & 25,16 \\
\hline std dev [ \pm CP] & 0,39 & 0,38 & 0,55 & 0,53 & 0,71 & 0,96 & 0,82 \\
\hline $\mathrm{CV}[\% \mathrm{CP}]$ & 1,82 & 2,71 & 2,32 & 2,89 & 3,97 & 5,09 & 3,51 \\
\hline $\min [x$-fold $]$ & $-1,67$ & $-1,99$ & $-1,86$ & $-2,35$ & $-2,17$ & $-3,14$ & $-2,34$ \\
\hline $\max [x$-fold $]$ & 1,93 & 1,58 & 2,71 & 2,21 & 6,27 & 6,34 & 3,27 \\
\hline std dev $[ \pm \mathrm{X}$-fold $]$ & 1,29 & 1,29 & 1,43 & 1,42 & 1,60 & 1,88 & 1,71 \\
\hline
\end{tabular}

Pearson correlation coefficient $(r)$

$\begin{array}{rccccccc}\text { VS. } & \text { HKG 1 } & \text { HKG 2 } & \text { HKG 3 } & \text { HKG 4 } & \text { HKG 5 } & \text { HKG 6 } & \text { HKG 7 } \\ \text { HKG 2 } & \mathbf{0 , 7 6 1} & - & - & - & - & - & - \\ \text { p-value } & \mathbf{0 , 0 0 7} & - & - & - & - & - & - \\ \text { HKG 3 } & \mathbf{0 , 6 7 3} & \mathbf{0 , 5 3 7} & - & - & - & - & - \\ \text { p-value } & \mathbf{0 , 0 2 3} & \mathbf{0 , 0 8 8} & - & - & - & - & - \\ \text { HKG 4 } & \mathbf{0 , 5 1 2} & \mathbf{0 , 6 1 0} & \mathbf{0 , 6 3 6} & - & - & - & - \\ \text { p-value } & \mathbf{0 , 1 0 7} & \mathbf{0 , 0 4 6} & \mathbf{0 , 0 3 6} & - & - & - & - \\ \text { HKG 5 } & -\mathbf{0 , 2 9 4} & -0,476 & -0,081 & -0,367 & - & - & - \\ \text { p-value } & \mathbf{0 , 3 8 2} & \mathbf{0 , 1 4 0} & \mathbf{0 , 8 1 6} & \mathbf{0 , 2 6 8} & - & - & - \\ \text { HKG 6 } & \mathbf{0 , 8 5 7} & \mathbf{0 , 4 6 1} & \mathbf{0 , 6 8 8} & \mathbf{0 , 5 2 8} & -0,189 & - & - \\ \text { p-value } & \mathbf{0 , 0 0 1} & \mathbf{0 , 1 5 3} & \mathbf{0 , 0 1 9} & \mathbf{0 , 0 9 4} & \mathbf{0 , 5 7 6} & - & - \\ \text { HKG 7 } & \mathbf{0 , 6 0 8} & \mathbf{0 , 3 3 8} & \mathbf{0 , 6 7 0} & \mathbf{0 , 3 1 7} & -\mathbf{0 , 4 2 8} & \mathbf{0 , 7 7 4} & - \\ \text { p-value } & \mathbf{0 , 0 4 7} & \mathbf{0 , 3 0 8} & \mathbf{0 , 0 2 4} & \mathbf{0 , 3 4 3} & \mathbf{0 , 1 8 9} & \mathbf{0 , 0 0 5} & - \\ \text { eper vs. } & \text { HKG 1 } & \text { HKG 2 } & \text { HKG 3 } & \text { HKG 4 } & \text { HKG 5 } & \text { HKG 6 } & \text { HKG 7 } \\ \text { corr. [r] } & \mathbf{0 , 8 7 0} & \mathbf{0 , 6 1 7} & \mathbf{0 , 8 7 8} & \mathbf{0 , 6 6 0} & -0,092 & 0,923 & 0,717 \\ \text { p-value } & \mathbf{0 , 0 0 1} & \mathbf{0 , 0 4 3} & \mathbf{0 , 0 0 1} & \mathbf{0 , 0 2 7} & \mathbf{0 , 7 8 6} & \mathbf{0 , 0 0 1} & \mathbf{0 , 0 1 3}\end{array}$

Regression Analysis: HKG vs. BestKeeper

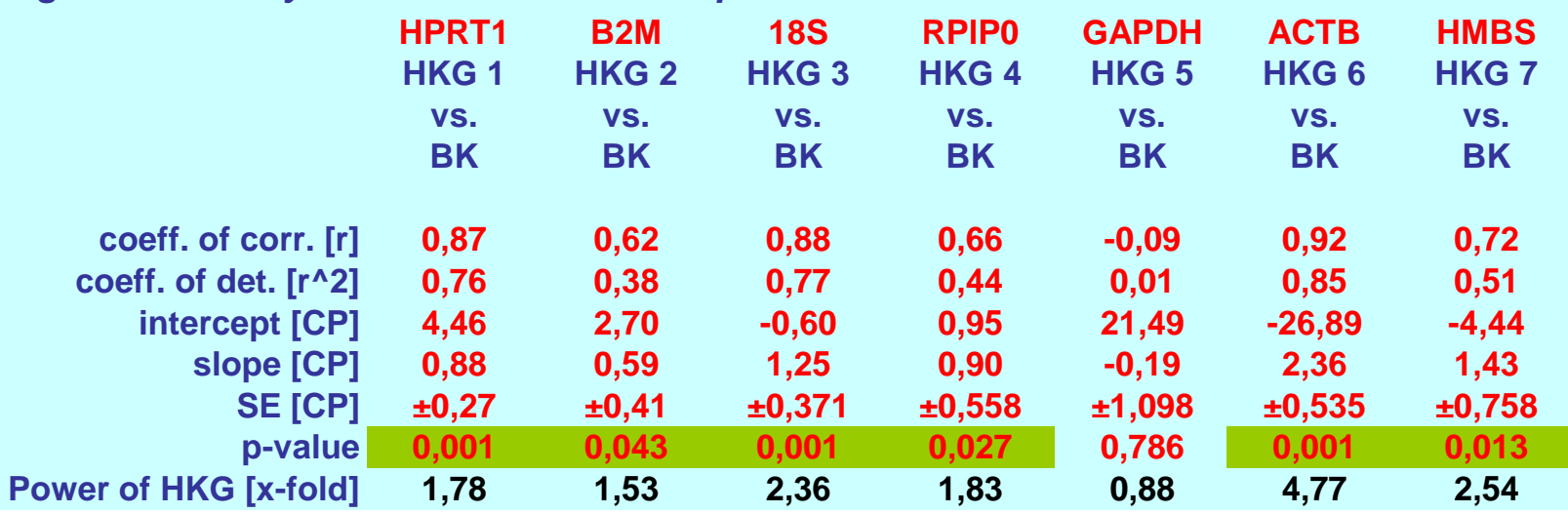


small intestine

\begin{tabular}{|c|c|c|c|c|c|c|c|}
\hline \multicolumn{8}{|c|}{ CP data of housekeeping Genes: } \\
\hline & HPRT1 & B2M & $18 S$ & RPIPO & GAPDH & АСТВ & HMBS \\
\hline & HKG 1 & HKG 2 & HKG 3 & HKG 4 & HKG 5 & HKG 6 & HKG 7 \\
\hline $\mathbf{n}$ & 12 & 12 & 12 & 12 & 12 & 12 & 12 \\
\hline geo Mean [CP] & 25,41 & 16,59 & 20,03 & 19,06 & 21,25 & 21,48 & 29,66 \\
\hline ar Mean $[\mathrm{CP}]$ & 25,42 & 16,64 & 20,05 & 19,07 & 21,29 & 21,53 & 29,69 \\
\hline $\min [C P]$ & 23,73 & 14,02 & 18,86 & 18,14 & 18,85 & 18,22 & 27,24 \\
\hline $\max [C P]$ & 26,75 & 18,58 & 21,52 & 20,42 & 23,89 & 23,60 & 31,44 \\
\hline std dev [ \pm CP] & 0,82 & 1,00 & 0,72 & 0,59 & 1,10 & 1,14 & 0,97 \\
\hline CV [\% CP] & 3,22 & 6,01 & 3,60 & 3,10 & 5,15 & 5,29 & 3,25 \\
\hline $\min [x-$ fold] & $-3,01$ & $-6,37$ & $-2,24$ & $-1,86$ & $-5,29$ & $-8,68$ & $-4,85$ \\
\hline $\max [x$-fold $]$ & 2,41 & 4,16 & 2,79 & 2,50 & 6,24 & 4,09 & 3,18 \\
\hline std dev [ \pm X-fold $]$ & 1,71 & 1,93 & 1,61 & 1,47 & 2,06 & 2,12 & 1,89 \\
\hline
\end{tabular}

Pearson correlation coefficient $(r)$

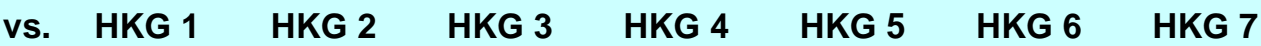

$\begin{array}{rccccccc}\text { HKG 2 } & \mathbf{0 , 7 5 9} & - & - & - & - & - & - \\ \text { p-value } & \mathbf{0 , 0 0 4} & - & - & - & - & - & - \\ \text { HKG 3 } & \mathbf{0 , 8 9 6} & \mathbf{0 , 8 4 5} & - & - & - & - & - \\ \text { p-value } & \mathbf{0 , 0 0 1} & \mathbf{0 , 0 0 1} & - & - & - & - & - \\ \text { HKG 4 } & \mathbf{0 , 9 1 1} & \mathbf{0 , 8 7 2} & \mathbf{0 , 9 2 1} & - & - & - & - \\ \text { p-value } & \mathbf{0 , 0 0 1} & \mathbf{0 , 0 0 1} & \mathbf{0 , 0 0 1} & - & - & - & - \\ \text { HKG 5 } & \mathbf{0 , 6 8 2} & \mathbf{0 , 3 9 0} & \mathbf{0 , 5 5 3} & \mathbf{0 , 6 4 2} & - & - & - \\ \text { p-value } & \mathbf{0 , 0 1 5} & \mathbf{0 , 2 1 0} & \mathbf{0 , 0 6 2} & \mathbf{0 , 0 2 4} & - & - & - \\ \text { HKG 6 } & \mathbf{0 , 8 4 9} & \mathbf{0 , 9 0 3} & \mathbf{0 , 8 9 6} & \mathbf{0 , 8 7 6} & \mathbf{0 , 4 1 9} & - & - \\ \text { p-value } & \mathbf{0 , 0 0 1} & \mathbf{0 , 0 0 1} & \mathbf{0 , 0 0 1} & \mathbf{0 , 0 0 1} & \mathbf{0 , 1 7 5} & - & - \\ \text { HKG 7 } & \mathbf{0 , 8 9 1} & \mathbf{0 , 7 4 1} & \mathbf{0 , 8 7 3} & \mathbf{0 , 8 6 5} & \mathbf{0 , 6 7 0} & \mathbf{0 , 8 5 9} & - \\ \text { p-value } & \mathbf{0 , 0 0 1} & \mathbf{0 , 0 0 6} & \mathbf{0 , 0 0 1} & \mathbf{0 , 0 0 1} & \mathbf{0 , 0 1 7} & \mathbf{0 , 0 0 1} & - \\ \text { eper vs. } & \text { HKG 1 } & \text { HKG 2 } & \text { HKG 3 } & \text { HKG 4 } & \text { HKG 5 } & \text { HKG 6 } & \text { HKG 7 } \\ \text { corr. [r] } & \mathbf{0 , 9 4 0} & \mathbf{0 , 8 9 4} & \mathbf{0 , 9 4 6} & \mathbf{0 , 9 6 4} & \mathbf{0 , 6 8 2} & \mathbf{0 , 9 3 1} & \mathbf{0 , 9 2 8} \\ \text { p-value } & \mathbf{0 , 0 0 1} & \mathbf{0 , 0 0 1} & \mathbf{0 , 0 0 1} & \mathbf{0 , 0 0 1} & \mathbf{0 , 0 1 5} & \mathbf{0 , 0 0 1} & \mathbf{0 , 0 0 1}\end{array}$

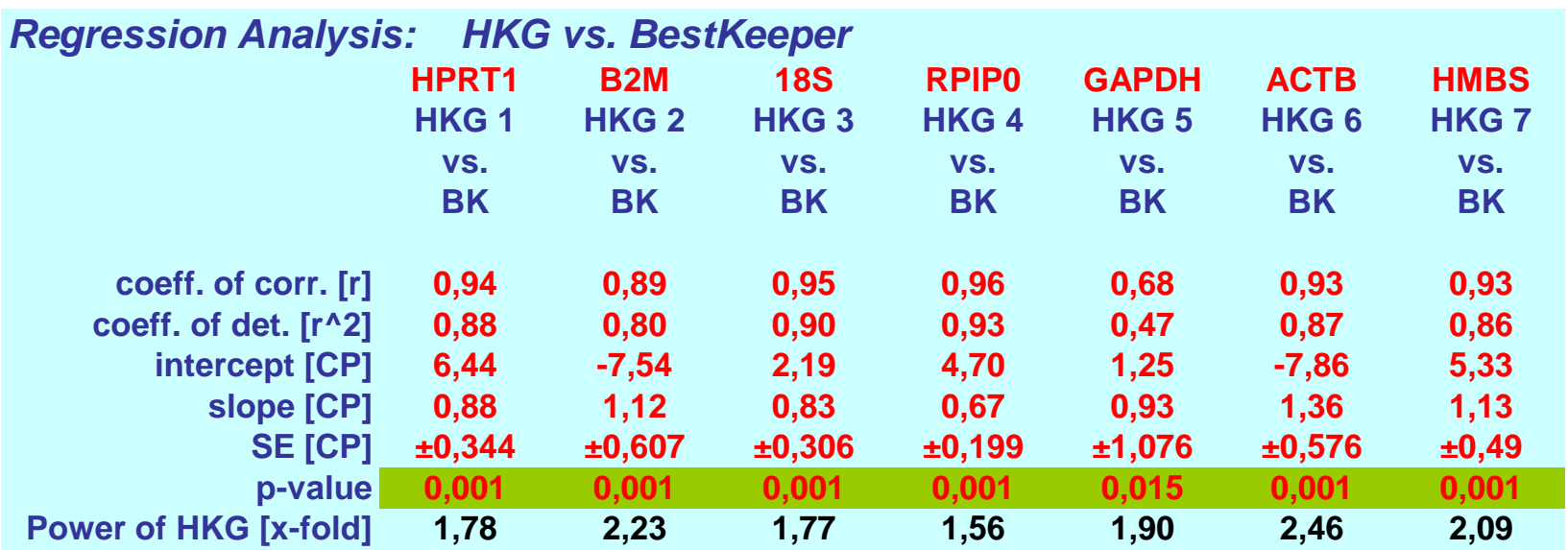




\section{Candidate reference genes for miRNA normalization}

\section{BestKeeper version 1}

http://gene-quantification.com/bestkeeper.html

liver

\begin{tabular}{|c|c|c|c|c|c|c|c|c|c|c|}
\hline \multicolumn{11}{|c|}{ CP data of housekeeping Genes: } \\
\hline & mir186 & mir142 & U6 & sno202 & miR16 & mir122 & sno234 & mir143 & mir19a & mir200a \\
\hline & HKG 1 & HKG 2 & HKG 3 & HKG 4 & HKG 5 & HKG 6 & HKG 7 & HKG 8 & HKG 9 & HKG 10 \\
\hline $\mathbf{n}$ & 12 & 12 & 12 & 12 & 12 & 12 & 12 & 12 & 12 & 12 \\
\hline geo Mean $[\mathrm{CP}]$ & 26,03 & 31,28 & 16,37 & 23,35 & 23,03 & 18,56 & 16,64 & 21,80 & 25,23 & 25,64 \\
\hline ar Mean [CP] & 26,03 & 31,28 & 16,38 & 23,35 & 23,03 & 18,56 & 16,64 & 21,80 & 25,23 & 25,65 \\
\hline $\min [\mathrm{CP}]$ & 25,64 & 30,40 & 15,15 & 22,68 & 22,56 & 17,97 & 16,13 & 21,23 & 24,66 & 24,21 \\
\hline $\max [\mathrm{CP}]$ & 26,49 & 32,55 & 17,14 & 24,25 & 23,40 & 19,03 & 17,20 & 22,46 & 25,86 & 26,62 \\
\hline std dev [ \pm CP] & 0,28 & 0,54 & 0,41 & 0,49 & 0,18 & 0,34 & 0,24 & 0,34 & 0,31 & 0,62 \\
\hline CV [\% CP] & 1,06 & 1,73 & 2,50 & 2,10 & 0,80 & 1,85 & 1,46 & 1,56 & 1,22 & 2,41 \\
\hline $\min [x$-fold] & $-1,31$ & $-1,83$ & $-2,34$ & $-1,59$ & $-1,39$ & $-1,50$ & $-1,42$ & $-1,49$ & $-1,48$ & $-2,69$ \\
\hline $\max [x-f o l d]$ & 1,38 & 2,42 & 1,70 & 1,87 & 1,29 & 1,39 & 1,48 & 1,57 & 1,55 & 1,97 \\
\hline std dev [ $\pm x$-fold] & 1,21 & 1,46 & 1,33 & 1,41 & 1,14 & 1,27 & 1,18 & 1,27 & 1,24 & 1,53 \\
\hline
\end{tabular}

\section{Pearson correlation coefficient ( $r$ )}

vs. HKG 1

HKG 20,338

p-value $\quad 0,281$

HKG $3 \quad 0,154$

p-value $\quad 0,635$

HKG $4 \quad 0,020$

p-value $\quad 0,953$

HKG $5 \quad-0,071$

p-value $\quad 0,823$

HKG

p-v

p-value $\quad 0,899$

HKG $7 \quad 0,183$

p-value $\quad 0,568$

HKG $8 \quad 0,394$

p-value $\quad 0,204$

0,717

0,009

$-0,335$

0,289

$-0,534$

HKG $9 \quad-0,308$

0,025

$-0,307$

0,938

0,332

$0,653 \quad 0,619$

$0,021 \quad 0,032$

0,676

0,016

$-0,367 \quad-0,204$

0,265

$-0,200$

$0,240 \quad 0,524$

0,736

0,006

$0,707 \quad 0,399$

$-0,227$

0,530

p-value $\quad 0,332$

0,010

0,198

0,476

0,381

0,223

0,095

0,770

0,429

0,404

$0,676 \quad 0,683$

0,165

0,192

HKG $10 \quad 0,025$

0,584

0,552

$-0,681$

$-0,356$

$0,015 \quad 0,258$

$-0,190$

0,555

0,383

0,219

$-0,372$

p-value $\quad 0,938$

$0,047 \quad 0,063$

0,621

0,233

0,066

0,777

0,838

0,355

0,003

0,262

0,260

0,415

$-0,381$

BestKeeper vs. HKG 1

HKG 2 HKG 3

HKG 4

HKG 5

HKG 6

HKG 7

HKG 8 0,223

coeff. of corr. [r]

0,331

0,844

0,007

$-0,067$

0,158

0,781

0,071

0,629

HKG 9 HKG 10 p-value 0,838

0,621

0,823

0,028

0,326

0,525

0,080

\section{Regression Analysis: HKG vs. BestKeeper}

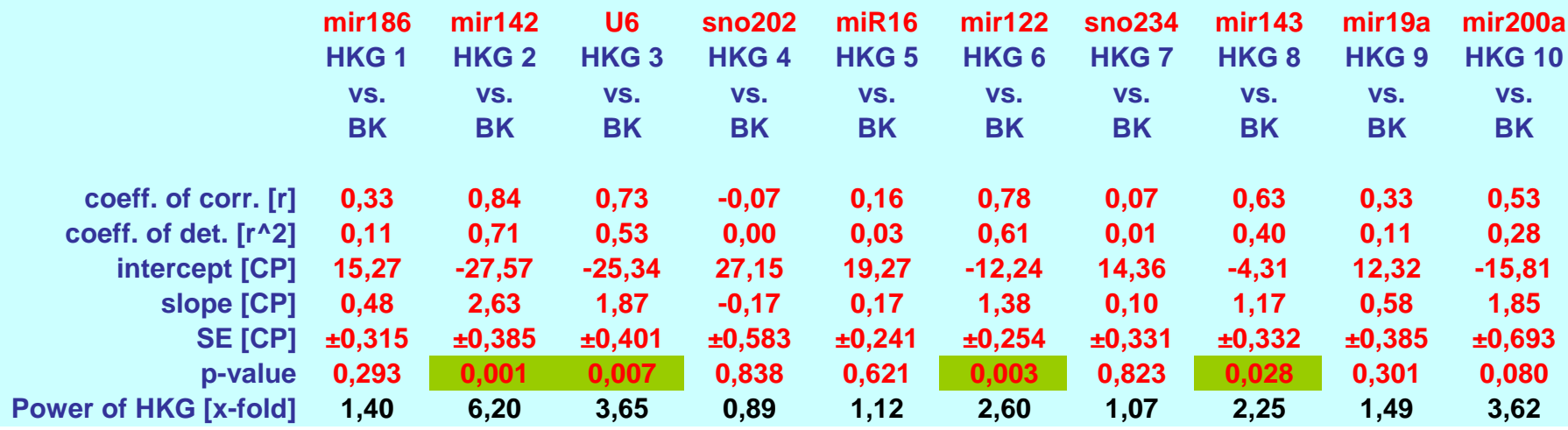


small intestine

\begin{tabular}{|c|c|c|c|c|c|c|c|c|c|c|}
\hline \multicolumn{11}{|c|}{ CP data of housekeeping Genes: } \\
\hline & mir186 & mir142 & U6 & sno202 & miR16 & mir122 & sno234 & mir143 & mir19a & mir200a \\
\hline & HKG 1 & HKG 2 & HKG 3 & HKG 4 & HKG 5 & HKG 6 & HKG 7 & HKG 8 & HKG 9 & HKG 10 \\
\hline $\mathbf{n}$ & 12 & 12 & 12 & 12 & 12 & 12 & 12 & 12 & 12 & 12 \\
\hline geo Mean $[\mathrm{CP}]$ & 24,15 & 29,48 & 15,97 & 17,88 & 19,77 & 28,82 & 16,71 & 17,31 & 23,41 & 19,31 \\
\hline ar Mean [CP] & 24,16 & 29,50 & 15,98 & 17,88 & 19,79 & 28,90 & 16,72 & 17,34 & 23,41 & 19,32 \\
\hline $\min [\mathrm{CP}]$ & 22,73 & 27,85 & 15,19 & 17,47 & 18,60 & 24,16 & 16,10 & 15,03 & 22,51 & 18,33 \\
\hline $\max [\mathrm{CP}]$ & 25,09 & 31,52 & 17,15 & 18,58 & 21,46 & 32,95 & 17,65 & 18,38 & 24,75 & 20,27 \\
\hline std dev [ $\pm \mathrm{CP}]$ & 0,44 & 0,84 & 0,44 & 0,34 & 0,69 & 1,55 & 0,32 & 0,65 & 0,51 & 0,44 \\
\hline CV [\% CP] & 1,82 & 2,85 & 2,72 & 1,91 & 3,48 & 5,35 & 1,88 & 3,75 & 2,17 & 2,28 \\
\hline $\min [x$-fold] & $-2,67$ & $-3,11$ & $-1,72$ & $-1,33$ & $-2,25$ & $-25,26$ & $-1,53$ & $-4,87$ & $-1,86$ & $-1,97$ \\
\hline $\max [x-f o l d]$ & 1,92 & 4,10 & 2,27 & 1,62 & 3,21 & 17,46 & 1,92 & 2,09 & 2,54 & 1,94 \\
\hline std dev [ $\pm \mathrm{X}$-fold] & 1,36 & 1,79 & 1,35 & 1,27 & 1,61 & 2,92 & 1,24 & 1,57 & 1,42 & 1,36 \\
\hline
\end{tabular}

\section{Pearson correlation coefficient $(r)$}

$\begin{array}{rcccccccccc}\text { vs. } & \text { HKG 1 } & \text { HKG 2 } & \text { HKG 3 } & \text { HKG 4 } & \text { HKG 5 } & \text { HKG 6 } & \text { HKG 7 } & \text { HKG 8 } & \text { HKG 9 } & \text { HKG } 10 \\ \text { HKG 2 } & \mathbf{0 , 7 2 6} & - & - & - & - & - & - & - & - & - \\ \text { p-value } & \mathbf{0 , 0 0 7} & - & - & - & - & - & - & - & - & - \\ \text { HKG 3 } & -0,396 & -0,124 & - & - & - & - & - & - & - & - \\ \text { p-value } & \mathbf{0 , 2 0 4} & \mathbf{0 , 6 9 8} & - & - & - & - & - & - & - & - \\ \text { HKG 4 } & -0,173 & \mathbf{0 , 0 7 3} & \mathbf{0 , 7 6 7} & - & - & - & - & - & - & - \\ \text { p-value } & \mathbf{0 , 5 8 8} & \mathbf{0 , 8 2 3} & \mathbf{0 , 0 0 4} & - & - & - & - & - & - & - \\ \text { HKG 5 } & \mathbf{0 , 1 6 5} & \mathbf{0 , 3 0 7} & \mathbf{0 , 6 4 6} & \mathbf{0 , 6 6 4} & - & - & - & - & - & - \\ \text { p-value } & \mathbf{0 , 6 0 8} & \mathbf{0 , 3 3 2} & \mathbf{0 , 0 2 3} & \mathbf{0 , 0 1 8} & - & - & - & - & - & - \\ \text { HKG 6 } & -0,213 & -0,098 & \mathbf{0 , 2 1 6} & \mathbf{0 , 0 0 2} & -0,373 & - & - & - & - & - \\ \text { p-value } & \mathbf{0 , 5 0 6} & \mathbf{0 , 7 6 3} & \mathbf{0 , 5 0 0} & \mathbf{0 , 9 9 2} & \mathbf{0 , 2 3 3} & - & - & - & - & - \\ \text { HKG 7 } & \mathbf{0 , 8 2 2} & \mathbf{0 , 8 5 2} & -\mathbf{0 , 3 0 7} & -\mathbf{0 , 1 5 9} & \mathbf{0 , 2 4 5} & -\mathbf{0 , 3 4 3} & - & - & - & - \\ \text { p-value } & \mathbf{0 , 0 0 1} & \mathbf{0 , 0 0 1} & \mathbf{0 , 3 3 2} & \mathbf{0 , 6 2 1} & \mathbf{0 , 4 4 2} & \mathbf{0 , 2 7 7} & - & - & - & - \\ \text { HKG 8 } & \mathbf{0 , 9 1 1} & \mathbf{0 , 6 0 7} & -\mathbf{0 , 4 9 7} & -\mathbf{0 , 3 2 9} & -0,050 & \mathbf{0 , 0 1 3} & \mathbf{0 , 6 7 4} & - & - & - \\ \text { p-value } & \mathbf{0 , 0 0 1} & \mathbf{0 , 0 3 7} & \mathbf{0 , 1 0 0} & \mathbf{0 , 2 9 7} & \mathbf{0 , 8 7 6} & \mathbf{0 , 9 6 9} & \mathbf{0 , 0 1 6} & - & - & - \\ \text { HKG 9 } & \mathbf{0 , 7 6 4} & \mathbf{0 , 6 9 0} & \mathbf{- 0 , 3 9 6} & \mathbf{- 0 , 2 7 2} & \mathbf{0 , 0 6 3} & -\mathbf{0 , 2 8 6} & \mathbf{0 , 7 8 5} & \mathbf{0 , 6 0 8} & - & - \\ \text { p-value } & \mathbf{0 , 0 0 4} & \mathbf{0 , 0 1 3} & \mathbf{0 , 2 0 4} & \mathbf{0 , 3 9 4} & \mathbf{0 , 8 4 5} & \mathbf{0 , 3 6 9} & \mathbf{0 , 0 0 2} & \mathbf{0 , 0 3 6} & - & - \\ \text { HKG 10 } & \mathbf{0 , 8 4 8} & \mathbf{0 , 6 3 8} & -0,340 & -0,225 & \mathbf{0 , 1 1 4} & -0,071 & \mathbf{0 , 6 9 2} & \mathbf{0 , 8 2 6} & \mathbf{0 , 7 5 9} & - \\ \text { p-value } & \mathbf{0 , 0 0 1} & \mathbf{0 , 0 2 6} & \mathbf{0 , 2 8 1} & \mathbf{0 , 4 8 2} & \mathbf{0 , 7 2 6} & \mathbf{0 , 8 2 3} & \mathbf{0 , 0 1 3} & \mathbf{0 , 0 0 1} & \mathbf{0 , 0 0 4} & - \\ & & & & & & & & & & \\ \text { BestKeeper vs. } & \text { HKG 1 } & \text { HKG 2 } & \text { HKG 3 } & \text { HKG 4 } & \text { HKG 5 } & \text { HKG 6 } & \text { HKG 7 } & \text { HKG 8 } & \text { HKG 9 } & \text { HKG 10 } \\ \text { coeff. of corr. [r] } & \mathbf{0 , 7 6 1} & \mathbf{0 , 8 0 2} & \mathbf{0 , 1 3 9} & \mathbf{0 , 2 2 0} & \mathbf{0 , 4 0 1} & \mathbf{0 , 2 2 7} & \mathbf{0 , 6 6 9} & \mathbf{0 , 7 1 1} & \mathbf{0 , 5 6 9} & \mathbf{0 , 7 5 0} \\ \text { p-value } & \mathbf{0 , 0 0 4} & \mathbf{0 , 0 0 2} & \mathbf{0 , 6 6 9} & \mathbf{0 , 4 9 4} & \mathbf{0 , 1 9 8} & \mathbf{0 , 4 7 6} & \mathbf{0 , 0 1 7} & \mathbf{0 , 0 0 9} & \mathbf{0 , 0 5 3} & \mathbf{0 , 0 0 5}\end{array}$

Regression Analysis: HKG vs. BestKeeper

mir186 mir142 U6

vs. vs. vs

BK BK BK BK

coeff. of det. [ $\left.\mathrm{r}^{\wedge} 2\right] \quad 0,58$

intercept [CP] $-1,1$

0,80

0,64

0,14

0,02

$-17,29$

11,73

slope [CP] 1,22

2,25

0,20

SE [CP] $\pm 0,421 \quad \pm 0,68$

$\pm 0,59$

p-value 0,004

0,002

0,669

1,15

0,22
0,05
13,07
0,23
$\pm 0,416$
0,494

1,17 vs. vs.

BK BK

0,40

0,16

0,85

0,91

$\pm 0,845$

0,198

1,88

0,23
0,05
2,22
1,28
$\pm 2,231$
0,476
2,43

0,23

2,22

2,43 sno234 mir143 mir19a mir200a

HKG 7 HKG 8 HKG 9 HKG 10

vs. vs. vs. vs.

BK BK BK BK

$\begin{array}{llll}0,67 & 0,71 & 0,57 & 0,75\end{array}$

$\begin{array}{llll}0,45 & 0,51 & 0,32 & 0,56\end{array}$

$1,34 \quad-17,21 \quad 3,39 \quad-4,69$

$\begin{array}{llll}0,74 & 1,66 & 0,96 & 1,15\end{array}$

$\pm 0,333 \quad \pm 0,666 \quad \pm 0,564 \quad \pm 0,413$

\begin{tabular}{ll|l|l|}
0,017 & 0,009 & 0,053 & 0,005
\end{tabular}

$\begin{array}{llll}1,67 & 3,16 & 1,95 & 2,23\end{array}$ 